\title{
Influência da idade e do contexto socioeducacional na compreensão emocional de crianças
}

\author{
Age and socio-educational effects on children's \\ emotional understanding
}

\author{
Pollyana de Lucena MOREIRA' \\ Eloá Losano de ABREU \\ Júlio RIQUE NETO'
}

\begin{abstract}
Resumo
O presente estudo teve o objetivo de verificar a influência da idade e do contexto socioeducacional na compreensão emocional de crianças. Participaram 117 crianças, com idade de 3 a 8 anos. As crianças foram divididas por tipos de escola (pública e privada) e por grupos de idade ( 3 anos, 4 a 5 anos e 6 a 8 anos). Como instrumento para a coleta de dados, foi utilizado o Test of Emotion Comprehension. Os resultados mostraram que as crianças de escolas privadas apresentaram maiores frequências de acerto no teste de compreensão das emoções do que aquelas de escolas públicas. Verificaram-se também maiores frequências de acerto no teste com o aumento da idade.
\end{abstract}

Unitermos: Desenvolvimento infantil. Emoções. Teste de compreensão emocional.

\begin{abstract}
This study examined the influence of socioeconomic contexts and age on children's emotional understanding. One hundred and seventeen children ranging in age from 3 to 8 years participated in the study. They were divided by type of school: public and private, and age: 3 years, ages 4-5years, and ages 6-8years. The Test of Emotion Comprehension was used for data collection. Results showed the influence of age and socioeconomic contexts on children's emotion understanding. Children from private schools obtained higher scores in Test of Emotion Comprehension than children from public schools. In addition there was higher frequency of correct answer in the test as children's age increased.
\end{abstract}

Uniterms: Childhood development. Emotion. Test of emotion comprehension.

A compreensão das emoções é uma área de estudos da Psicologia que busca entender a relação entre as emoções e o desenvolvimento de habilidades sociais, ajudando as crianças a se adaptarem socialmente e promovendo sua saúde mental. A suposição básica é a de que uma melhor compreensão das emoções permite uma melhor regulação emocional e facilita a resolução de conflitos interpessoais desde a infância.

Os objetivos deste estudo foram verificar a compreensão emocional em dois grupos de crianças na

$\cot$

- Universidade Federal da Paraíba, Centro de Ciências Humanas, Letras e Artes, Departamento de Psicologia. Cidade Universitária, Castelo Branco, 58051-900, João Pessoa, PB, Brasil. Correspondência para/Correspondence to: P.L. MOREIRA. E-mail: <pollyanadelucena@gmail.com>.

Apoio: Conselho Nacional de Desenvolvimento Científico e Tecnológico (Processo n 575284/2008-0). 
faixa etária de 3 a 8 anos, bem como a influência do contexto socioeducacional nessa compreensão. A compreensão das emoções foi definida operacionalmente como a capacidade de reconhecer e compreender, no Eu e nos Outros, as emoções, suas causas e possibilidades de controle (Pons, Harris \& De Rosnay, 2004). O contexto socioeducacional foi observado a partir do tipo de escola (pública ou privada) em que as crianças estavam inseridas. Na cultura brasileira, esses dois tipos de escolas atendem a crianças advindas de níveis sociais diferentes e, consequentemente, adotam diferentes formas de socialização.

Pons et al. (2004), pioneiros na literatura especializada, estudaram o desenvolvimento da compreensão emocional em grupos de crianças de 3 a 11 anos, observando que esse processo se inicia a partir dos 3 anos de idade. Os autores identificaram nove componentes relacionados com a compreensão das emoções: Reconhecimento, Causas Externas, Desejo, Crença, Lembrança, Regulação, Mascaramento, Ambivalência e Moralidade. Pons e Harris (2005) organizaram esses nove componentes da compreensão emocional por níveis de dificuldade. Cada nível é composto por três componentes, do componente mais fácil ao mais difícil. A primeira etapa de desenvolvimento da compreensão emocional ocorre com a criança focando nos aspectos externos das emoções e no impacto que esses eventos têm a partir de sua lembrança. Posteriormente, a criança foca nos aspectos psicológicos das emoções, como o papel exercido pelos desejos e crenças e a distinção entre emoções sentidas e emoções expressadas. Finalmente, a criança reflete sobre as emoções e passa a entendê-las a partir de diferentes perspectivas, utilizando estratégias metacognitivas para o controle de pensamentos e, ainda, focando nos aspectos morais das emoções (Quadro 1).

Pons et al. (2004) sugeriram que o avanço na hierarquia dos componentes, do mais fácil para o mais difícil, não está necessariamente relacionado com a idade em que esses componentes se tornam acessíveis para as crianças. O avanço é verificado pela forma como eles são acessados e processados pelas crianças. Como mostra o Quadro 1, alguns dos componentes são mais difíceis que outros, dentro de uma mesma faixa etária. Por exemplo, o Desejo, um componente mental de dificuldade média pela teoria, ocorre entre os 3 e 5 anos de idade, a mesma faixa etária em que ocorre a Lembrança, que é um componente considerado de fácil aquisição por focar nos elementos externos da situação.

De Rosnay, Harris e Pons (2008) constataram que a habilidade de tomada de perspectiva social pode ser considerada como um antecedente necessário para o desenvolvimento da compreensão emocional. É a partir da tomada de perspectiva que as crianças se encontram hábeis para predizer as reações emocionais e comportamentais nos outros, em diferentes situações. Por exemplo, a criança já pode ser capaz de perceber que suas crenças e desejos são diferentes das crenças e desejos dos outros (Dunn \& Herrera, 1997). A tomada de perspectiva social passa a ser utilizada de forma mais complexa quando as crianças tentam resolver conflitos em situações ambivalentes, ou que necessitem de um controle das emoções ou que envolvam dilemas morais.

Quadro 1. Descrição dos componentes da compreensão emocional.

\begin{tabular}{|c|c|c|c|}
\hline & Nível & Componente (Idade) & Definição \\
\hline & \multirow[t]{3}{*}{ Externo/Fácil } & Reconhecimento (3-4) & Reconhece e nomeia emoções básicas. \\
\hline & & Lembranças (3-6) & Entende o efeito das lembranças nas emoções. \\
\hline & & Causas Externas (3-4) & Entende o efeito de causas externas sobre as emoções. \\
\hline & \multirow[t]{3}{*}{ Mental/Intermediário } & Crenças (4-6) & Entende o efeito das crenças, falsas ou verdadeiras, sobre as emoções. \\
\hline 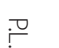 & & Desejo (3-5) & Entende o efeito de seus desejos nas reações emocionais dos outros. \\
\hline ב⿳亠口冋口 & & Mascaramento (4-6) & $\begin{array}{l}\text { Entende que existe uma discrepância entre sentimentos vividos e sentimentos } \\
\text { expressos. }\end{array}$ \\
\hline $\begin{array}{l}\prod_{D}^{D} \\
\text { D } \\
\end{array}$ & \multirow[t]{3}{*}{ Reflexivo/Difícil } & Moralidade (+/- 8) & $\begin{array}{l}\text { Entende que as emoções são associadas a comportamentos ou ações sociais e } \\
\text { morais. }\end{array}$ \\
\hline & & Regulação (6-7) & Cria estratégias comportamentais para manter o controle das emoções. \\
\hline & & Ambivalência (+/-8) & Entende que é possível sentir emoções mistas diante de algumas situações. \\
\hline
\end{tabular}

762

Fonte: Pons, Harris \& Rosnay (2004). 
O modo como o desenvolvimento da compreensão emocional ocorre é associado às diferenças individuais. Várias pesquisas verificaram essa associação com a qualidade do apego que as crianças possuem com os pais, com o tipo de socialização parental e com o contexto socioeconômico em que estão inseridas. Por exemplo, Pons, Doudin, Harris e De Rosnay (2005) sugerem que o tipo de vínculo que as crianças possuem com os pais está relacionado com o modo como elas resolvem seus conflitos emocionais. Conforme os autores, as crianças que possuem fortes vínculos com os pais possuem uma autorregulação mais eficaz do que as crianças que não formam esse tipo de vínculo. Os autores mencionam ainda que o tipo de socialização utilizada pelos pais relaciona-se com o desenvolvimento da compreensão das emoções. O desenvolvimento ocorre na medida em que, em uma determinada situação, os pais explicam às crianças as causas das emoções, desejos, crenças e intenções, e as consequências de comportamentos, que podem estar direcionadas tanto para elas próprias como para outras pessoas.

Conger e Donnellan (2007) estudaram fatores sociais e econômicos que influenciam o desenvolvimento das crianças em diferentes níveis socioeconômicos. Eles explicam que, em famílias com menor condição financeira, os pais preocupam-se principalmente em suprir suas necessidades econômicas e, consequentemente, apresentam-se mais autoritários e punitivos, não explicando aos filhos as consequências dos comportamentos adotados por ambos, pais e filhos. Essas características inibem o desenvolvimento de competências sociais e emocionais nas crianças e diminuem o suporte afetivo de que elas precisam para um desenvolvimento emocional saudável.

Por outro lado, Conger e Donnellan (2007) afirmam que pais com uma situação financeira satisfatória preocupam-se mais com o desenvolvimento dos filhos, estimulam a aprendizagem, oferecem as melhores escolas, usam vocabulário rico e promovem estimulações cognitivas que fazem com que eles pensem nas consequências de seu comportamento. Portanto, do ponto de vista desses autores, quanto melhor a condição econômica da família, maior a probabilidade de haver uma melhor qualidade no desenvolvimento da compreensão emocional.

Corroborando a ideia de que fatores socioeconômicos influenciam o desenvolvimento da compreen- são emocional, alguns estudos foram realizados em outras culturas, como os de Tenenbaum, Visscher, Pons e Harris (2004) e de Roazzi, Dias, Minervino, Roazzi e Pons (2009). Os resultados mostraram diferenças em relação à organização dos componentes, quando comparados com os resultados obtidos em estudos anteriores, através do Test of Emotion Comprehension (TEC) (Pons \& Harris, 2000; Pons et al., 2004).

Tenenbaum et al. (2004) realizaram estudo com crianças da tribo peruana quéchua, utilizando uma versão adaptada do TEC para a região. Participaram da pesquisa 39 crianças com idade de 4 a 11 anos, divididas em dois grupos (4-7 anos e 8-11 anos), sendo verificado que as mais velhas entenderam melhor os componentes do TEC. Quando comparadas com as crianças inglesas (amostra de Pons et al. 2004), verificou-se que as crianças da tribo quéchua apresentaram uma organização diferente dos componentes e mostraram menor frequência de acerto. Como um dos resultados mais interessantes do estudo, os autores apontaram o fato de as Causas Externas começarem a ser entendidas a partir dos 8 anos de idade, resultado atribuído à dificuldade de acesso dessas crianças à educação formal (moravam em uma vila agrária e pastoril) e à falta de explicação e estimulação dos adultos.

Um estudo transcultural para a validação do TEC em países de língua portuguesa foi realizado no Brasil por Roazzi, Dias, Minervino, Roazzi \& Pons (2009). Participaram 396 crianças dos Estados de Pernambuco, Paraíba e Rio Grande do Norte, com idade de 4 a 12 anos, divididas em oito faixas etárias e dois tipos de escola: públicas e privadas. Os resultados indicaram que, para essa amostra, a organização dos componentes diferiu da organização proposta pela teoria, como foi verificado no estudo de Tenenbaum et al. (2004).

Considerando o contexto socioeconômico (escola pública ou privada) e a idade das crianças, o estudo de Roazzi et al. (2009) revelou que as provenientes de escolas privadas apresentaram maior frequência de acerto no TEC do que as de escolas públicas. Mais uma vez, esse resultado vem indicar a influência do contexto socioeducacional e/ou econômico no desenvolvimento da compreensão emocional.

Seguindo os autores acima citados, o presente estudo propõe investigar a influencia da idade e dos contextos educacionais no desenvolvimento da compreensão emocional em crianças de idades e grupos 
diferentes dos utilizados por Roazzi et al. (2009). Ainda, os procedimentos adotados para este estudo seguiram as orientações originais de correção do instrumento TEC. Assim, torna-se possível comparar os resultados encontrados neste estudo com os resultados encontrados em outras culturas (exemplo: quéchua), como mais uma forma de verificar a universalidade da teoria e sua aplicabilidade ao contexto brasileiro.

\section{Método}

Este trabalho tem um cunho correlacional, buscando verificar a influência da idade e do contexto socioeconômico e educacional na compreensão emocional de crianças.

\section{Participantes}

Participaram 117 crianças, com idade média de 4,37 anos ( $D P=1,45)$, sendo 50 crianças de escolas privadas e 67 crianças de escolas públicas da cidade de João Pessoa, na Paraíba. As crianças foram divididas em três grupos de idade, da seguinte forma: 32 crianças de 3 anos, 68 crianças de 4 a 5 anos, e 17 crianças de 6 a 8 anos.

\section{Instrumentos}

Teste de Compreensão Emocional (Pons \& Harris, 2000). Consiste em um livro de gravuras, que acessa os nove componentes da compreensão emocional em duas versões, uma para meninos e outra para meninas. Cada versão apresenta um total de 23 cenários pictóricos, encontrados na parte superior das páginas do instrumento. Os cenários são distribuídos por componentes, da seguinte forma: Reconhecimento (5 cenários), Causas Externas (5 cenários), Desejo (2 cenários), Crença (1 cenário), Lembrança (4 cenários), Regulação (1 cenário), Mascaramento (1 cenário), Ambivalência (1 cenário) e - Moralidade (3 cenários). Para cada cenário existe um 3. script que conta uma estória. Em seguida, são apresentadas quatro gravuras, representando emoções que variam de acordo com a estória. Para cada estória existe apenas uma emoção correta. A atribuição de escores é feita da seguinte forma: o pesquisador atribui 1 ponto para cada componente respondido corretamente, e 0 (zero) para cada componente que a criança errou.
Questionário Biodemográfico. É um questionário objetivo, no qual o pesquisador preenche informações sobre as crianças: idade, gênero e nível educacional.

\section{Procedimentos}

O estudo foi submetido ao Comitê de Ética e Pesquisa da Universidade Federal da Paraíba (Protocolo n 0167), tendo atendido a todos os requisitos éticos da Resolução no 196/96. Após obter a permissão das escolas para a realização da pesquisa, foram enviados envelopes com o Termo de Consentimento Livre e Esclarecido, informando aos pais a finalidade da pesquisa e pedindo sua autorização para que o filho pudesse participar da atividade. Apenas as crianças autorizadas foram entrevistadas.

Procedimento de administração do Teste de Compreensão Emocional. A administração do instrumento foi feita individualmente, com o pesquisador lendo uma estória para cada criança, apontando para o cenário e perguntando: "Como você acha que o personagem $X$ está se sentindo?". A criança era solicitada a apontar a emoção mais apropriada para a estória contada. Cada entrevista teve uma duração aproximada de 20 minutos, e as respostas de cada criança foram anotadas em uma folha de respostas que acompanhava o instrumento.

Procedimento de análise dos dados do Teste de Compreensão Emocional. As informações coletadas por meio do TEC foram passadas para um banco de dados do Statistical Package for the Social Sciences (SPSS), versão 16, para análises. As análises consideraram os tipos de escolas (pública e privada) e os grupos de idade (3 anos, 4 a 5 anos e 6 a 8 anos). Inicialmente, foram analisadas as frequências de acerto das crianças por componente, considerando o tipo de escola e os grupos de idade. Posteriormente, foram efetuados testes de Kruskal-Wallis para verificar a existência de diferenças entre os escores doTEC por tipos de escola e entre os escores do TEC por grupos de idade. Também foi realizada uma Análise de Variância (Anova) para verificar o efeito do tipo de escola e da idade nos escores do TEC.

\section{Resultados}

A Tabela 1 mostra as frequências de acerto das crianças por componente e por tipo de escola. Pode-se verificar, exceto nos componentes Crença e Regulação, 
que as crianças das escolas privadas obtiveram maior frequência de acerto dos componentes do que as crianças das escolas públicas. A análise de Kruskal-Wallis revelou que existe uma diferença significativa entre os escores do TEC e o tipo de escola $\left(\chi_{\mathrm{kw}(1)}^{2}=4,485 ; p=0,028\right)$.

Considerando os grupos de idade, como pode ser observado na Tabela 2, houve um aumento da frequência de acerto das crianças por componentes, com o aumento da idade. As crianças de 6 a 8 anos obtiveram maior frequência de acertos do que as crianças de 3 anos e as de 4 a 5 anos. Essa tendência de maior frequência de acerto com o aumento da idade só não foi observada nos componentes Reconhecimento e Ambivalência. Para esses componentes, a maior frequência de acertos concentrou-se nas crianças de 4 a 5 anos. A análise de Kruskal-Wallis demonstrou que existe uma diferença significativa entre os escores do TEC e os grupos de idade $\left(\chi_{\mathrm{Kw}(1)}^{2}=10,39 ; p=0,0006\right)$.

Tabela 1. Comparação das frequências de acerto das crianças por componente, considerando o tipo de escola. João Pessoa (PB), 2010.

\begin{tabular}{|c|c|c|c|c|}
\hline \multirow{2}{*}{ Componentes } & \multicolumn{2}{|c|}{ Escola pública $(n=67)$} & \multicolumn{2}{|c|}{ Escola particular $(n=50)$} \\
\hline & $\mathrm{F}$ & $\%$ & $F$ & $\%$ \\
\hline Reconhecimento & 17 & 25,4 & 23 & 46 \\
\hline Causas Externas & 1 & 1,5 & 2 & 4 \\
\hline Desejo & 14 & 20,9 & 14 & 28 \\
\hline Crença & 24 & 35,8 & 16 & 32 \\
\hline Lembrança & 24 & 35,8 & 27 & 54 \\
\hline Regulação & 12 & 17,9 & 13 & 26 \\
\hline Mascaramento & 26 & 38,8 & 16 & 32 \\
\hline Ambivalência & 17 & 25,4 & 16 & 32 \\
\hline Moralidade & 5 & 7,5 & 7 & 14 \\
\hline
\end{tabular}

Tabela 2. Frequências de acerto das crianças por componentes, considerando a idade. João Pessoa (PB), 2010.

\begin{tabular}{|c|c|c|c|c|c|c|}
\hline \multirow{3}{*}{ Componente } & \multicolumn{6}{|c|}{ Grupo de idade } \\
\hline & \multicolumn{2}{|c|}{3 anos $(n=32)$} & \multicolumn{2}{|c|}{$4-5$ anos $(n=68)$} & \multicolumn{2}{|c|}{$6-8$ anos $(n=17)$} \\
\hline & $F$ & $\%$ & $\mathrm{~F}$ & $\%$ & $F$ & $\%$ \\
\hline Reconhecimento & 6 & 18,0 & 22 & 33,8 & 11 & 6,7 \\
\hline Causas Externas & 1 & 3,1 & 1 & 1,5 & 1 & 5,9 \\
\hline Desejo & 2 & 6,3 & 15 & 22,1 & 11 & 64,7 \\
\hline Crença & 7 & 21,9 & 25 & 36,8 & 8 & 47,1 \\
\hline Lembrança & 11 & 34,4 & 30 & 44,1 & 10 & 58,8 \\
\hline Regulação & 6 & 18,8 & 13 & 19,1 & 6 & 35,3 \\
\hline Mascaramento & 7 & 21,9 & 26 & 38,2 & 9 & 52,9 \\
\hline Ambivalência & 9 & 28,1 & 23 & 33,8 & 1 & 5,9 \\
\hline Moralidade & 3 & 9,4 & 7 & 10,3 & 2 & 11,8 \\
\hline
\end{tabular}

Tabela 3. Médias de acerto nos componentes, considerando o tipo de escola e a idade. João Pessoa (PB), 2010.

\begin{tabular}{|c|c|c|c|c|c|}
\hline \multirow{2}{*}{ Componentes } & \multicolumn{2}{|c|}{ Tipo de escola } & \multicolumn{3}{|c|}{ Grupo de escola } \\
\hline & Pública (n=67) & Particular $(n=50)$ & 3 anos $(n=32)$ & $4-5$ anos $(n=68)$ & $6-8$ anos $(n=17)$ \\
\hline Reconhecimento & $0,25(0,44)$ & $0,46(0,50)$ & $0,19(0,39)$ & $0,34(0,48)$ & $0,65(0,49)$ \\
\hline Causas externas & $0,01(0,12)$ & $0,04(0,19)$ & $0,03(0,18)$ & $0,01(0,12)$ & $0,06(0,24)$ \\
\hline Desejo & $0,21(0,41)$ & $0,28(0,54)$ & $0,06(0,24)$ & $0,22(0,42)$ & $0,65(0,49)$ \\
\hline Crença & $0,36(0,48)$ & $0,32(0,47)$ & $0,22(0,42)$ & $0,37(0,48)$ & $0,47(0,51)$ \\
\hline Lembrança & $0,36(0,48)$ & $0,54(0,50)$ & $0,34(0,48)$ & $0,44(0,50)$ & $0,59(0,35)$ \\
\hline Regulação & $0,18(0,38)$ & $0,26(0,44)$ & $0,19(0,39)$ & $0,19(0,39)$ & $0,35(0,49)$ \\
\hline Mascaramento & $0,39(0,49)$ & $0,32(0,47)$ & $0,22(0,42)$ & $0,38(0,49)$ & $0,53(0,51)$ \\
\hline Ambivalência & $0,25(0,44)$ & $0,32(0,47)$ & $0,28(0,46)$ & $0,34(0,47)$ & $0,06(0,24)$ \\
\hline Moralidade & $0,07(0,26)$ & $0,14(0,35)$ & $0,09(0,29)$ & $0,10(0,30)$ & $0,12(0,33)$ \\
\hline
\end{tabular}


A Tabela 3 mostra as médias de acerto das crianças por componentes, considerando o tipo de escola e os grupos de idade. Através de uma Anova 3×2 (grupos de idade: 3 anos, 4 a 5 anos e 6 a 8 anos versus tipo de escola: pública e privada), tendo os escores do TEC como variáveis dependentes, verificou-se um efeito significativo do tipo de escola $\left[F_{(1,112)}=11,790, p<0,001\right]$ e dos grupos de idade $\left[F_{(2,112)}=9,933, p<0,001\right]$ nos escores do TEC. Ou seja, a Anova verificou que tanto o tipo de escola como a idade são fatores que influenciam os acertos das crianças no TEC e, consequentemente, influenciam o desenvolvimento da compreensão emocional.

Considerando os níveis de dificuldade propostos pela teoria, seja por tipo de escola, seja por grupo de idade, verificou-se que o desenvolvimento não correspondeu ao esperado. Componentes classificados como fáceis pela teoria, como, por exemplo, Causas Externas, obtiveram baixas frequências de acerto, enquanto componentes classificados pela teoria como de nível intermediário, como, por exemplo, Mascaramento, obtiveram altas frequências de acerto.

\section{Discussão}

A teoria da Compreensão Emocional foi desenvolvida a partir de estudos realizados com crianças de cultura europeia, revelando como elas desenvolvem o entendimento das emoções. É esperado que ocorra uma ordem de dificuldade na apreensão dos componentes, o que reflete um processo de interiorização do mundo externo, com incremento da capacidade de assimilações reflexivas pautadas em elementos psicológicos, como a diferenciação entre o Outro e o Eu nas abstrações mentais. Na análise do desenvolvimento nos componentes, observou-se um aumento da frequência de acerto no TEC com o avanço da idade, nas crianças tanto de escolas públicas quanto privadas.

Por outro lado, no tocante à ordem de dificuldade dos componentes, estudos estão encontrando variações que são atribuídas a contextos socioeconômicos e educacionais. Tanto na comparação por idade quanto na comparação por tipo de escola, as frequências de acertos mostraram os componentes fáceis da teoria sendo classificados como difíceis, enquanto componentes intermediários e difíceis da teoria apre- sentaram maior facilidade. Merecem destaque os resultados relacionados com os componentes Ambivalência, Reconhecimento e Causas Externas.

Classificada pela teoria como difícil, a Ambivalência apresentou a segunda maior frequência de acertos das crianças de 3 anos, havendo uma queda progressiva dos acertos com o avanço da idade. O Reconhecimento, classificado como fácil pela teoria, apresentou a maior frequência de acertos apenas para as crianças de 6 a 8 anos, sugerindo uma tendência à estabilização do componente a partir dos 6 anos de idade. Com relação ao tipo de escola, nesse mesmo componente, apenas as crianças das escolas privadas apresentaram altas frequências de acertos. Já o componente Causas Externas, classificado como fácil pela teoria, apresentou as frequências de acertos mais baixas, tanto na comparação por idade como na comparação por escola. Esse resultado corrobora outras pesquisas similares (Roazzi et al., 2009; Tenenbaum et al., 2004) nas duas condições socioeconômicas estudadas.

A comparação dos resultados entre as crianças de escolas públicas e privadas mostrou que as primeiras apresentaram maior dificuldade (ou menor frequência de acertos) ao responder ao TEC do que as crianças de escolas privadas. Esse resultado apoia a afirmação de Conger e Donnellan (2007) de que crianças provenientes de um contexto social mais favorecido possuem melhor suporte afetivo de seus pais e maiores chances de ter um desenvolvimento emocional adequado.

Por outro lado, é plausível perguntar se o TEC apresenta itens que sejam representativos das diversas culturas empiricamente estudadas. Roazzi et al. (2009) já alertaram para essa questão, no sentido que consideraram que alguns itens são caracteristicamente mais europeus que brasileiros. Considerando essa observação, é possível teorizar que famílias de classes economicamente favorecidas no Brasil valorizem uma educação no estilo europeu, em contraste com famílias que lutam pela sobrevivência econômica. Considerou-se, porém, que não é possível afirmar a influência do estilo de socialização parental na compreensão emocional das crianças sem ter verificado essa variável; assim, esse aspecto apresentou-se como uma limitação deste estudo.

É importante salientar que determinados aspectos, como o número de itens por componentes e a habilidade de tomada de perspectiva social das crian- 
ças, podem ter influenciado os resultados encontrados. No TEC, os componentes não são avaliados pelo mesmo número de itens. Por exemplo, componentes como Reconhecimento e Causas Externas possuem cinco itens, enquanto outros componentes, como Mascaramento e Ambivalência, possuem apenas um item. Dessa forma, a probabilidade de uma criança acertar um componente com um ou dois itens é maior do que a probabilidade de acertar um componente com cinco itens. Além disso, para serem compreendidos, todos os componentes necessitam da capacidade de tomada de perspectiva social, alguns de forma mais simples, outros de forma mais complexa. Nesse sentido, se as crianças não têm essa capacidade desenvolvida ou em processo de desenvolvimento, o entendimento adequado das emoções ou de situações que envolvem conflitos emocionais pode ficar comprometido, influenciando, assim, os escores do TEC.

\section{Considerações Finais}

Em conclusão, as limitações do presente estudo apresentam-se em torno de duas questões: as práticas de socialização parental e a habilidade de tomada de perspectiva social.

As práticas de socialização parental devem ser consideradas para que se possa atribuir diferenças no desenvolvimento da compreensão emocional. Como apresentado na revisão da literatura, o tipo de socialização parental, relacionado às questões financeiras da família, influencia o modo como a compreensão das emoções se desenvolve nas crianças. Nesse sentido, a ausência de investigação acerca das práticas de socialização parental experimentadas pelas crianças deste estudo, impediu de saber até que ponto elas influenciam o desenvolvimento da compreensão emocional e como essa influência ocorre.

Outro ponto a ser considerado é a relação entre a compreensão emocional e a tomada de perspectiva social, avaliadas no TEC. Os itens do TEC avaliam o desenvolvimento da compreensão emocional a partir da habilidade de tomada de perspectiva social, ou seja, solicita-se que as crianças tomem a perspectiva de um personagem e indiquem a emoção que ele está sentindo em dada situação. Os itens relacionados com os componentes considerados difíceis exigem uma tomada de perspectiva social mais elaborada do que aqueles itens relacionados com componentes considerados fáceis.
Portanto, o fato de não se ter investigado a tomada de perspectiva social neste estudo não permitiu verificar se a variação nessa habilidade cognitiva teria influenciado os escores no TEC, e o modo como a compreensão das emoções estaria se desenvolvendo.

Por último, para adaptação e a construção da versão em português do TEC, é preciso considerar que os resultados possam estar sendo influenciados pelo número desigual de itens entre os componentes e, ainda, pela possibilidade de algumas respostas não representarem adequadamente as diversas culturas existentes no Brasil.

\section{Referências}

Conger, R. D., \& Donnellan, M. B. (2007). An interactionist perspective on the socioeconomic context of human development. Annual Review of Psychology, 58, 175-199.

De Rosnay, M., Harris, P. L., \& Pons, F. (2008). Making links between emotion understanding and developmental psychopathology in young children: normative patterns and the case of maltreatment. In C. Sharp, P. Fonagy \& I. Goodyer (Eds.), Social cognition and developmental psychopathology. New York: Oxford University Press.

Dunn, J., \& Herrera, C. (1997). Conflict resolution with friends, siblings and mothers: a developmental perspective. Aggressive Behavior, 23 (5), 343-357.

Pons, F., Doudin, P. A., Harris, P. L., \& de Rosnay, M. (2005). Helping children to improve their emotion comprehension. In F. Pons, D. R. Hancock, L. Lafortune \& P. A. Doudin (Orgs.), Emotions in Learning (pp.15-39). Deumark: Aalborg University Press.

Pons, F., \& Harris, P. L. (2000). Test of Emotion Comprehension - TEC. Oxford: University of Oxford.

Pons, F., \& Harris, P. L. (2005). Longitudinal change and longitudinal stability of individual differences in children's emotion understanding. Cogniton and Emotion, 19 (8):1158-1174.

Pons, F., Harris, P. L., \& de Rosnay, M. (2004). Emotion understanding between 3 and 11 years: developmental periods and hierarchical organization. European Journal of Developmental Psychology, 1 (2),127-152.

Roazzi, A., Dias, M. B. B., Minervino, C. M., Roazzi, M., \& Pons, F. (2009). Children's Comprehension of Emotion: a crosscultural investigation. In D. Elizer \& E. Yaniv (Org), Theory construction and multivariade analysis: aplications of facet approach (pp.109-123). Israel: Bar-llan University.

Tenenbaum, H. R, Visscher, P., Pons, F. \& Harris, P. L. (2004). Emotion understanding in quechua children from an agro-pastoralist Village. International Journal of Behavioral Development, 28 (5), 471-478.

Recebido em: 1/8/2011

Versão final em: 4/6/2012

Aprovado em: 21/6/2012 
